\title{
Autographen und Manuskripte zur ETH- und zur Wissenschaftsgeschichte
}

Aus den Wissenschaftshistorischen Sammlungen der ETH-Bibliothek Zürich

Von Beat Glaus

Hauptaufgabe der Eidgenössischen Technischen Hochschule wie auch ihrer Bibliothek ist die - infolge Finanzklemme und Personalstopp schwierig, ja prekär gewordene - Bewältigung der aktuellen Aufgaben in Lehre und Forschung. Für die historische Dimension: die Geschichte der Schule, der Wissenschaft und der Technik, bleiben unter diesen Umständen nur wenig Mittel übrig. Dies trifft auch auf die Wissenschaftshistorischen Sammlungen der ETH-Bibliothek zu, welche deren Handschriften, Rara, Porträts und Biographische Dossiers verwalten. Ihre Erwerbung muß heute praktisch ohne Kredit auskommen - und ist deshalb noch mehr als früher auf kleinen und größeren - Geschenkeingang angewiesen.

Das C.G. Jung-Archiv

Carl Gustav Jung war bekanntlich auch Dozent für Psychologie an der ETH Zürich (1933-1941). Im Jubiläumsjahr 1955 wurde er hier zum «Doktor der Naturwissenschaften ehrenhalber» ernannt. 1977 schenkte die Erbengemeinschaft seinen wissenschaftlichen Nachlaß der Hochschule, die ihn den Wissenschaftshistorischen Sammlungen der ETH-Bibiliothek zuwies. Vollzogen wurde die Donation während der letzten zwei Jahre. 1980 konnte das über 30000 Autographen umfassende Briefkorpus übernommen werden. Es ist, in der überkommenen Ordnung, bereits benützbar - sofern die Normen des Urheberrechts und Persönlichkeitsschutzes eingehalten werden. Die archivarische Aufbereitung dieses Riesenwerks begann 1981 und wird, zusammen mit der Katalogisierung, noch Jahre in Anspruch nehmen, ja nur mit Hilfe des Computers zu vollenden sein. Im gleichen Jahre wurde die Schenkung im wesentlichen abgeschlossen, indem uns auch die OriginalManuskripte von Jungs publiziertem Lebenswerk anvertraut wurden. Im übrigen waren wir in der glücklichen Lage, dieses wichtige Forschungspo- 
tential durch «flankierende Maßnahmen» zu ergänzen; bereicherten doch bedeutende Persönlichkeiten und Institutionen aus der Schule Jungs das Archiv mit wissenschaftsgeschichtlichen Akten: So Professor Carl Alfred Meier, der Jungs ETH-Professur weiterführte (selber aber ohne Nachfolger blieb); ferner die Psychologin Aniela Jaffé (Jungs letzte Sekretärin), Frau Dr. Marie-Louise von Franz sowie der Psychologische Club (dieser mit dem Nachlaß Jolande Jacobis).

Trotz dem beträchtlichen Umfang weist dieses Archiv indessen eine spürbare Lücke auf: Das Briefkorpus umfaßt etwa zwei Drittel Briefe von $\mathrm{x}$ Absendern an C.G.Jung. Jungs eigene Briefe sind fast ausschließlich Typoskriptkopien (wohl meist von Hand der Sekretärin), und ein guter Teil derselben besteht aus Routineantworten. Was (naheliegenderweise) vor allem fehlt, sind zahlreiche der meist viel wesentlicheren Briefe von Jungs eigener Hand, die wirkliche Unikate darstellen und sich, aus begreiflicher Ursache, nach wie vor im Besitze der Empfänger befinden. Im Interesse künftiger Forschung wäre es wichtig, möglichst viele dieser inhaltlich hochbedeutsamen Dokumente durch Archivierung sicherzustellen und damit letzten Endes auch der Wissenschaft erst verfügbar zu machen. Aus praktischen Gründen (möglichst geringe Zersplitterung eines wichtigen Autographenschatzes) böte sich als zentrale Aufbewahrungsstätte hiefür wohl in erster Linie das C. G. Jung-Archiv der ETH-Bibliothek an! Geneigten Donatoren darf versichert werden, daß jede Ergänzung hier ebenso dankbar entgegengenommen wie sachkundig archiviert und erschlossen wird!

\section{Nachschriften von Vorlesungen hervorragender ETH-Dozenten}

Teilweise schon im letzten Jahrhundert war es am Eidgenössischen Polytechnikum üblich, daß für zentrale Vorlesungen Skripten angefertigt, maschinell vervielfältigt (z. B. lithographiert) und den Studenten abgegeben wurden. Die Wissenschaftshistorischen Sammlungen hüten unter ihren Raritäten mehrere «Frühdrucke», z. B.:

Albert Heim (1849-1937), Professor für Geologie an Universität und ETH Zürich 1837-1911): Allgemeine Geologie; Notizen aus der vierstündigen Vorlesung des Herrn Professors Dr. Albert Heim mit dessen gütiger Erlaubnis aufgezeichnet von Leon Wehrli, stud.phil. 2 Bde. Zürich 1892 (vervielfältigt durch den Verband der Polytechniker, s. Abb.). 


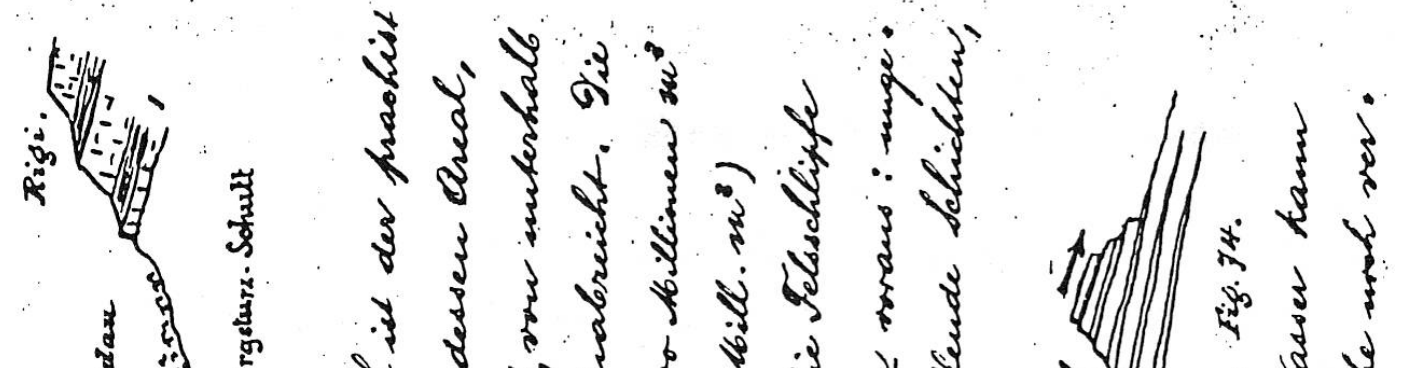
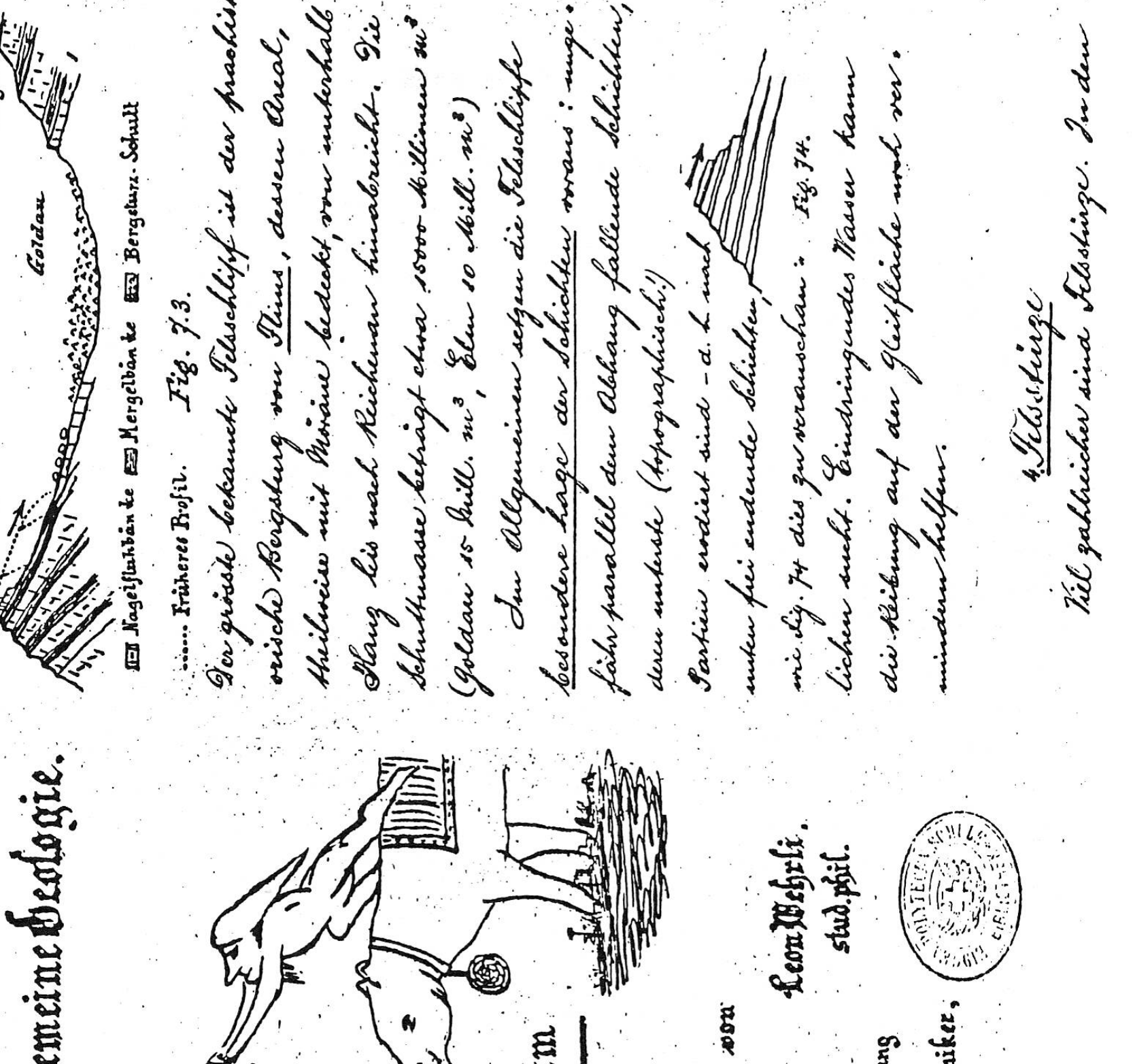
$\frac{1}{3} \sqrt{2}$

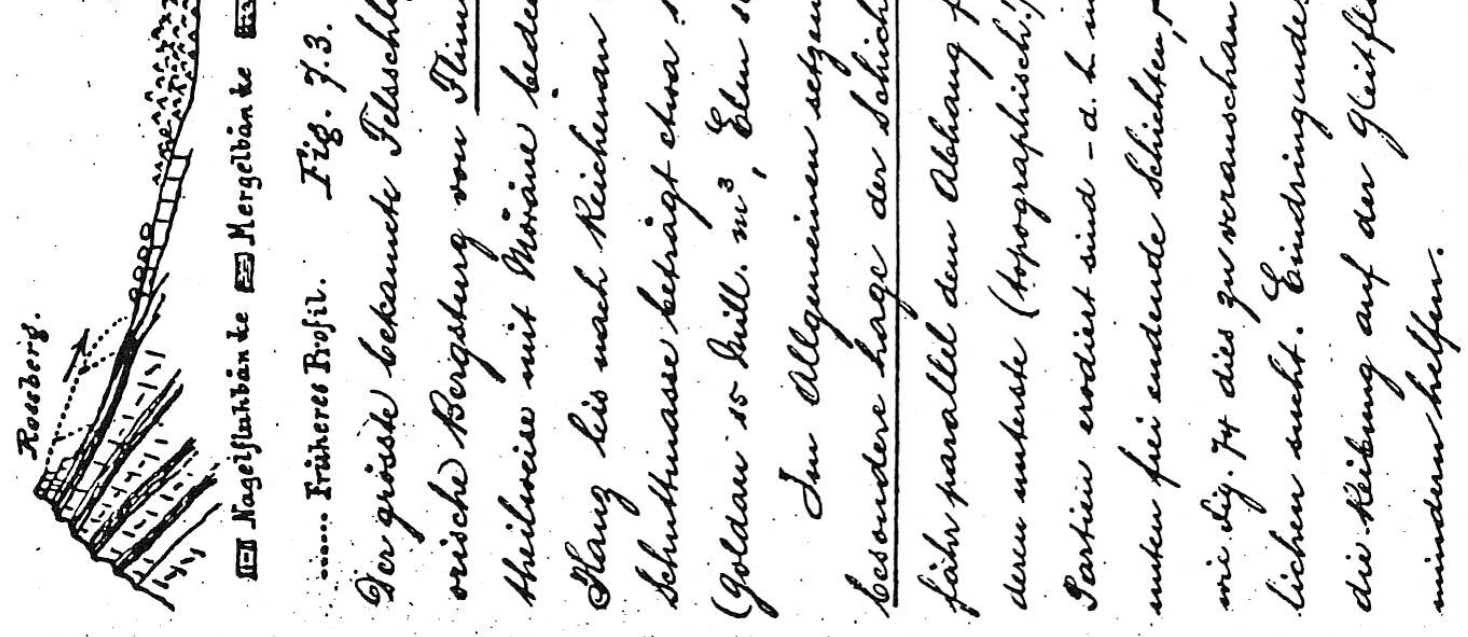

Da
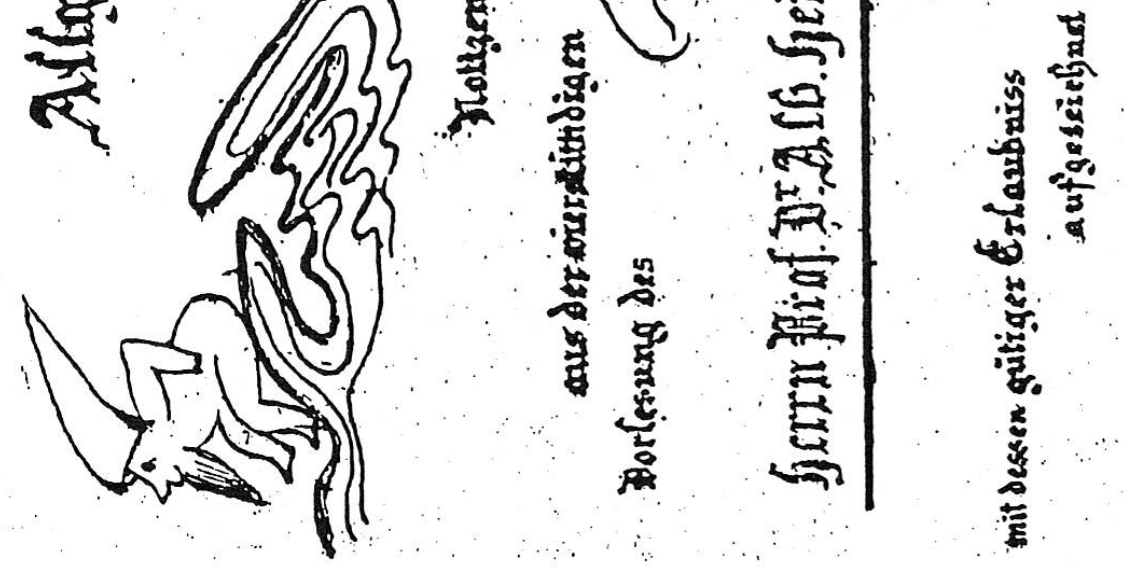

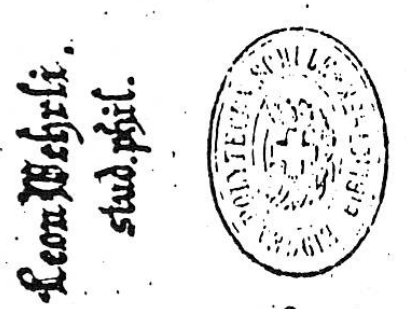

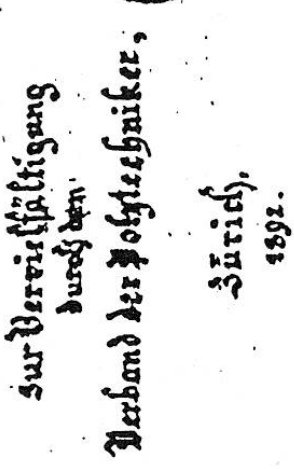


Ludwig Tetmajer (1850-1905, Professor für Baumechanik an der ETH 1878-1901, Direktor der Eidgenössischen Materialprüfungsanstalt): Schmiedeiserne Dächer. Als Manuskript autographiert. Zürich 1880. Georg Veith (1821-1903, Professor für Maschinenbaukunde und Maschinenkonstruieren an der ETH 1868-1893): Maschinenbau; Text und Skizzenbuch der Vorlesung für den 2. und 3. Mechanischen Kurs. 7 Bde. (Zürich: Eidgenössisches Polytechnikum 1879-1882).

Friedrich Weber (1843-1912); Professor für mathematische und technische Physik an der ETH 1875-1912): Ausarbeitung der Vorlesungen über Technische Physik von H.F. Weber; Polytechniker-Verein: Physik I-LIII. (Zürich: Eidgenössisches Polytechnikum, um 1880).

Heute werden solche Skripten recht häufig hergestellt und meist von den studentischen Fachvereinen gedruckt und vertrieben. Damit ist der den Studenten zu einer bestimmten Zeit vermittelte Lehr- und Lernstoff in der Regel auch für die später einsetzende historische Forschung gesichert, da Drucksachen den Weg in eine öffentliche Bibliothek normalerweise leicht finden und dort auch auf Dauer aufbewahrt werden. Von sehr vielen - und gerade auch hervorragenden - Vorlesungen wurden und werden indessen gar nie solche Vervielfältigungen angefertigt! Wenn der Wissenschaftshistoriker beispielweise wissen möchte, was Albert Einstein (1879-1955) denn während seiner drei Semester als Professor für theoretische Physik an der ETH doziert hat, ist er voll und ganz auf handschriftliche Quellen angewiesen. Unter diesen nehmen die persönlichen Nachschriften einzelner Studenten eine hervorragende Stellung ein, bewahren sie doch oft den während einer bestimmten Vorlesung vermittelten Stoff authentischer als die - gerne jahrlang gebrauchten und damit oft variierten - Notizen des Dozenten selber (vide Werner Kaegis diesbezüglich Lamentationes in seiner Biographie Jacob Burckhardts über dessen «Zettelwirtschaft»)! Einsteins Lehrtätigkeit an der ETH ist glücklicherweise in Nachschriften von «zwei Händen» überliefert: Der angehende Elektroingenieur Walter Dällenbach $\left({ }^{*} 1892\right)$ hielt sämtliche Vorlesungen in - allerdings knappen, teilweise stenographischen - Notizen fest. Von der zweistündigen Vorlesung «Statistische Mechanik» (SS 1913) fertigten V. Gorgé und O. Hänni aus dem Institut für theoretische Physik der Universität Bern, in Zusammenarbeit mit Herrn Dällenbach, eine vervielfältigte Umschrift an. (Sie kann bei der ETHBibliothek zum Preis von Fr. 15.- bezogen werden. Weitere Umschriften sind geplant.) Die Vorlesung über «Elektrizität und Magnetismus» aber ist 
zusätzlich in mustergültigem Stenogramm eines weiteren Hörers: des Maschineningenieurs Eduard Sidler $\left({ }^{*} 1889\right)$, erhalten. Der gleiche Mann überlieferte uns übrigens auch in gleich getreuer Weise die Vorlesungen, welche C.G. Jung an der ETH gehalten hat!

Bei vielen Dozenten, nicht zuletzt solchen unseres Jahrhunderts, welche sowohl für die Geschichte der ETH als auch für die Wissenschaftsgeschichte allgemein erstrangige Bedeutung besitzen, läßt die Dokumentation ihrer Vorlesungen jedoch noch sehr zu wünschen übrig. Von wichtigen Persönlichkeiten ist sie bei uns nur in «zufälligen» Einzelmanuskripten präsent, so z. B. von:

- Peter Debye (1884-1966; Professor für Physik an der ETH 1920-1927): Molekülbau; Kinetische Theorie der Aggregatzustände; Ionen und Elektronen. Vorlesungsnachschriften von Dipl. Ing. Emanuel Jaquet (18971981) aus den Jahren 1920-22;

- Paul Scherrer (1890-1969; Professor der Physik an der ETH 1920-1960): Experimentalphysik I (SS 1922); Nachschriften von stud.phil. Conrad Burri $\left({ }^{*} 1900\right)$ und stud.chem. Ernst Schüpbach $\left({ }^{*} 1902\right)$;

- Hermann Staudinger (1881-1965; Professor für Allgemeine Chemie an der ETH 1912-1926): Vorlesungen über Anorganische und Organische Chemie, 1920-1922; in Nachschrift von Max Brunner (*1901), stud. Abt.f. Fachlehrer in Naturwissenschaften.

Weit umfangreicher als das Dokumentierte aber ist die Desideratenliste der Wissenschaftshistorischen Sammlungen für das noch zu Dokumentierende. Beispielsweise fehlen in der ETH-Bibliothek vollständig solche persönlichen Vorlesungsnachschriften (um mit den Nobelpreisträgern zu beginnen) zu:

- Richard Kuhn (1900-1967; Professor für Allgemeine Chemie an der ETH 1926-1929);

- Wolfgang Pauli (1900-1958; Professor für Theoretische Physik 19281958);

- Vladimir Prelog (*1906; Professor für organische Chemie 1945-1976);

- Thadeus Reichstein (*1897, PD und Professor für organische Chemie an der ETH 1930-1938);

- Leopold Ruzicka (1887-1976; Professor für Allgemeine Chemie an der ETH 1929-1957);

- Richard Willstätter (1872-1942; Professor für Allgemeine Chemie an der ETH 1905-1912). 
Ganz zu schweigen von einer weit beträchtlicheren Anzahl ähnlich, aber auch weniger bedeutender Namen aus den verschiedensten Gebieten des Lehrangebotes unserer Hochschule! Dieses kann bei der heutigen Aktenlage nur sehr fragmentarisch und oberflächlich rekonstruiert werden, da dem Wissenschaftshistoriker für eine genauere Analyse dessen, was zu einer bestimmten Zeit in einem bestimmten Fach auch wirklich gelehrt und vorgetragen wurde, auf weiten Gebieten ebendiese Unterlagen noch fehlen! Wir würden uns glücklich schätzen, wenn wir auch dieses - je länger desto seltener werdende - Forschungsgut vervollständigen könnten!

\section{Summary}

The Collections for the History of Science are a small but precious part of the ETH-Bibliothek, the Main Library of the Swiss Federal Institute of Technology in Zurich. Among numerous lecture notes, scientific or biographic autographs and manuscripts, since 1980 they also contain the family archives of Carl Gustav Jung, the famous Psychiatrist (1875-1961). This important donation comprises the original drafts of Jung's works and his extensive correspondence. Nearly all of Jung's own letters are, however, typewritten carbon-copies only, and most of the handwritten ones are still in the possession of the recipients. For the benefit of future research and also for security reasons all owners of Jungian letters are strongly recommended to present these documents, in original or copy form, to the C.G.Jung-Archives of the ETHBibliothek, CH-8092 Zurich!

Dr. Beat Glaus

ETH-Bibliothek

Rämistraße 101

CH-8092 Zürich 